\title{
Coherent structures in the linearized impulse response of turbulent channel flow
}

\author{
Sabarish B. Vadarevu†, Simon J. Illingworth and Ivan Marusic \\ Department of Mechanical Engineering, The University of Melbourne, Parkville, VIC 3010, \\ Australia \\ (Received $\mathrm{xx}$; revised $\mathrm{xx}$; accepted $\mathrm{xx}$ )
}

\begin{abstract}
We study the evolution of velocity fluctuations due to an isolated spatio-temporal impulse using the linearized Navier-Stokes equations. The impulse is introduced as an external body force in incompressible channel flow at $R e_{\tau}=10000$. Velocity fluctuations are defined about the turbulent mean velocity profile. A turbulent eddy viscosity is added to the equations to fix the mean velocity as an exact solution, which also serves to model the dissipative effects of the background turbulence on large-scale fluctuations. An impulsive body force produces flowfields that evolve into coherent structures containing long streamwise velocity streaks that are flanked by quasi-streamwise vortices; some of these impulses produce hairpin vortices. As these vortex-streak structures evolve, they grow in size to be nominally self-similar geometrically with an aspect ratio (streamwise to wallnormal) of approximately 10, while their kinetic energy density decays monotonically. The topology of the vortex-streak structures is not sensitive to the location of the impulse, but is dependent on the direction of the impulsive body force. All of these vortex-streak structures are attached to the wall, and their Reynolds stresses collapse when scaled by distance from the wall, consistent with Townsend's attached eddy hypothesis.
\end{abstract}

\section{Introduction}

Linear processes play an essential role in the subcritical transition to turbulence (Henningson \& Reddy 1994, Waleffe 1995) and to the sustenance of turbulence (Kim \& Lim 2000) in wall-bounded shear flows. Linear analyses involve decomposing the velocity fields into a base flow and perturbations about the base flow; for transition to turbulence, the base flow is the steady laminar solution, while for fully turbulent flows, the appropriate choice for the base flow is the turbulent mean velocity Reynolds \& Tiederman 1967). The turbulent mean velocity is linearly stable, and the coupling term in the linearized Navier-Stokes equations (LNSE) is responsible for the sustenance of turbulence structures as shown by $\operatorname{Kim} \& \operatorname{Lim}(2000)$. In more recent work, McKeon \& Sharma (2010) exposed the nature of the linear processes to be that of selective amplification of certain modes (which are travelling waves in their analysis), which allows a substantial reduction in the dimensionality (number of degrees of freedom) of high Reynolds number turbulence (Moarref et al. 2013).

A turbulent eddy viscosity is often introduced to the LNSE to fix the turbulent mean velocity profile as an exact solution of the equations, and to model the dissipative effect of the small-scales of turbulence on the large-scale perturbations (Reynolds \& Hussain 1972). The eddy-viscosity-enhanced linearized Navier-Stokes equations (eLNSE) have been successful in describing several features of wall-bounded turbulence. For instance, Del Álamo \& Jimenez (2006) and Pujals et al. (2009) showed that dominant turbulence

$\dagger$ Email address for correspondence: sabarish.vadarevu@unimelb.edu.au 
structures are captured by the eLNSE as the perturbations with the largest transient growth; Hwang \& Cossu (2010) extended these to study the response to harmonic and stochastic forcing, and showed the existence of inner and outer peaks in spanwise size of maximally amplified structures, as well as a self-similar range between the two peaks. Hwang (2016) suggested that the eddy viscosity enhancement could also help reconcile the inner-outer interaction (Mathis et al. 2009) in the near-wall region. A review of LNSE and eLNSE models for wall-bounded turbulence has recently been presented by McKeon (2017).

The impulse response completely characterizes a linear dynamical system in the time domain. In the context of the linearized dynamics of turbulence, this involves studying the response to non-linearities that are localized in both space and time. Using the impulse response to study coherent structures has the advantage of parametrizing their evolution without the need to impose a priori either their wall-parallel dimensions (i.e. fundamental Fourier modes in the streamwise and spanwise directions) or a phase relationship between the Fourier modes that constitute these structures. Several researchers have applied the impulse response or similar tools to turbulent flows. Although not using the impulse response directly, Landahl (1990) argued that the near-wall dynamics of turbulence can be considered as the linear response to spatio-temporally localized non-linear terms because of the high intermittency of wall-normal velocity fluctuations. Jovanović \& Bamieh (2001) used the impulse response to study the evolution of perturbations about a laminar base flow. Luchini et al. (2006) did this for turbulent flows by introducing white noise at the wall in a DNS and computing space-time correlations at $R e_{\tau}=180$. Codrignani (2014) extended the DNS-based study to different wall-normal locations for the impulse at a slightly lower Reynolds number of $R e_{\tau}=150$. More recently, Eitel-Amor et al. (2015) looked at the evolution of hairpin vortices at a higher Reynolds number of $R e_{\tau}=$ 590 , using the eLNSE as well as DNS to include a fully turbulent background. They found that the evolution of a single hairpin vortex observed in the turbulent DNS is well represented by the eddy-viscosity-enhanced LNSE; however, the linear model failed to capture regeneration, consistent with the earlier observations of $\operatorname{Kim} \& \operatorname{Lim}(2000)$.

In this paper, the low computational cost of the eLNSE model is exploited to extend these investigations of the impulse response to turbulent flows at higher Reynolds numbers; we use $R e_{\tau}=10000$ to allow for a decade of wall-normal extent of the $\log$ layer, although computations at higher $R e_{\tau}$ are also feasible. The aims of the work are to investigate (i) if the impulse response captures the vortex-streak structure known to be important to turbulence (Jiménez \& Moin 1991, Hwang 2015), (ii) the prevalence of the hairpin vortex (Adrian 2007), and (iii) the geometrical scaling of the coherent structures and associated turbulent stresses as they evolve in time. The numerical formulation for computing the impulse response is presented in $\S 2$ and the results for nine different cases of impulses are shown in $\S 3$. In $\S 4$, we summarize the principal observations of this paper and discuss the limitations of the present model.

\section{Numerical formulation}

The flow under consideration is incompressible channel flow. The velocity field is decomposed as the turbulent mean and perturbations about this mean, $\mathbf{u}_{\text {total }}=$ $[U(z), 0,0]+\mathbf{u}$. A Cartesian coordinate system is employed, where $x, y$, and $z$ represent the streamwise, spanwise, and wall-normal directions respectively. The perturbation vector $\mathbf{u}$ has components $[u, v, w]^{T}$, where $u$ is the streamwise velocity, $v$ is the spanwise velocity, and $w$ is the wall-normal velocity. We are interested in the evolution of these perturbations about a specified turbulent mean. The turbulent mean, $U(z)$, is computed 
by integrating an integrand containing the turbulent eddy viscosity, $\left(-u_{\tau} R e_{\tau} \eta\right) /\left(\nu_{T} / \nu\right)$, from $\eta=-1$ to $z$. Here, $z$ is the wall-normal coordinate non-dimensionalized by channel half-height such that the channel walls are at $z= \pm 1, \eta$ is a proxy for the non-dimensionalized wall-normal coordinate, $R e_{\tau}$ is the Reynolds number based on the friction velocity $u_{\tau}$, and $\nu_{T}$ is the total eddy viscosity. The total eddy viscosity $\nu_{T}=\nu_{t}+\nu$ contains the turbulent eddy viscosity $\nu_{t}$ and molecular viscosity $\nu$, and is approximated using the semi-analytical expression of Cess (1958) as reported by Reynolds \& Tiederman (1967):

$$
\nu_{T}=\frac{\nu}{2}\left[1+\frac{\kappa^{2} R e_{\tau}^{2}}{9}\left(1-z^{2}\right)^{2}\left(1+2 z^{2}\right)^{2}\left\{1-\exp \left(\frac{R e_{\tau}(|z|-1)}{A}\right)\right\}^{2}\right]^{1 / 2}+\frac{\nu}{2}
$$

where values for the von Kármán constant $\kappa=0.426$, and the constant $A=25.4$ are set to fit the turbulent mean velocity profile at $R e_{\tau}=2000$ (Hoyas \& Jiménez 2006).

We investigate the evolution of perturbations due to a spatio-temporally impulsive body force, which is considered to represent an idealized bursting event. This spatiotemporal impulse, $\tilde{\delta}\left(\mathbf{x}-\mathbf{x}_{0}, t\right)$, is factorized into impulses along each direction and time as

$$
\tilde{\delta}\left(\mathbf{x}-\mathbf{x}_{0}, t\right)=\delta(x) \delta(y) \delta\left(z-z_{0}\right) \delta(t),
$$

where $\delta$ is the Dirac delta function. The impulse is introduced at $x=y=t=0$, with a variable wall-normal location $z_{0}$. The Fourier transform of the impulse along $x$ and $y$ produces a coefficient of unity for each Fourier mode.

A momentum impulse along the direction $\left(m_{x} \hat{\mathbf{e}}_{x}+m_{y} \hat{\mathbf{e}}_{y}+m_{z} \hat{\mathbf{e}}_{z}\right)$, $\hat{\mathbf{e}}_{s}$ being the unitvector along direction $s \in\{x, y, z\}$, enters the evolution equations for any Fourier mode $e^{i\left(k_{x} x+k_{y} y\right)}$ as follows.

$$
\begin{aligned}
\partial_{t} \hat{\boldsymbol{\phi}} & =A \hat{\boldsymbol{\phi}}+B \hat{\mathbf{f}} \delta(t), \\
\hat{\mathbf{u}} & =C \hat{\boldsymbol{\phi}},
\end{aligned}
$$

where the state $\hat{\boldsymbol{\phi}}=[\hat{w}, \hat{\eta}]^{T}$ contains the Fourier coefficients of wall-normal velocity and wall-normal vorticity, and $\hat{\mathbf{f}}(z)=\left[m_{x} \delta\left(z-z_{0}\right), m_{y} \delta\left(z-z_{0}\right), m_{z} \delta\left(z-z_{0}\right)\right]^{T}$ is the wavenumber-independent Fourier coefficient for the impulsive body force (which models an idealized bursting event in the present work). The operators $A, B$, and $C$ are dependent on the wavenumbers of the Fourier mode, and are presented below.

$$
\begin{gathered}
A=\left[\begin{array}{cc}
\Delta^{-1} \mathcal{L}_{O S} & 0 \\
-i k_{y} U^{\prime} & \mathcal{L}_{S Q}
\end{array}\right], \quad C=\frac{1}{k_{x}^{2}+k_{y}^{2}}\left[\begin{array}{cc}
i k_{x} \mathcal{D} & -i k_{y} \\
i k_{y} \mathcal{D} & i k_{x} \\
k_{x}^{2}+k_{y}^{2} & 0
\end{array}\right], \\
B=\left[\begin{array}{ccc}
-i k_{x} \Delta^{-1} \mathcal{D} & -i k_{y} \Delta^{-1} \mathcal{D} & -\left(k_{x}^{2}+k_{y}^{2}\right) \Delta^{-1} \\
i k_{y} & -i k_{x} & 0
\end{array}\right],
\end{gathered}
$$

where, the prime and $\mathcal{D}$ represent wall-normal differentiation, $\Delta=\mathcal{D}^{2}-\left(k_{x}^{2}+k_{y}^{2}\right)$, and the operators $\mathcal{L}_{O S}$ and $\mathcal{L}_{S Q}$ are

$$
\begin{aligned}
& \mathcal{L}_{O S}=i k_{x} U^{\prime \prime}-i k_{x} U \Delta+\nu_{T} \Delta^{2}+2 \nu_{T}^{\prime} \Delta \mathcal{D}+\nu_{T}^{\prime \prime}\left(\mathcal{D}^{2}+\left(k_{x}^{2}+k_{y}^{2}\right)\right), \\
& \mathcal{L}_{S Q}=-i k_{x} U+\nu_{T} \Delta+\nu_{T}^{\prime} \mathcal{D} .
\end{aligned}
$$

See chapter 10 of Jovanović (2004) for a similar investigation of perturbations about the laminar flow; the operators $B$ and $C$ remain the same as in that study, while the operator $A$ is identical to the one used in Hwang \& Cossu (2010) to reflect the linearization about the turbulent mean velocity and the introduction of the turbulent eddy viscosity.

The velocity perturbations for any Fourier mode at a time $t$ due to an impulse 
introduced at time $t=0$ is calculated explicitly for any time $t>0$, without resorting to time-marching, as

$$
\hat{\mathbf{u}}=C e^{A t} B \hat{\mathbf{f}},
$$

which is obtained by integrating eq. 2.3. The impulse response is computed for a spatially periodic flow with domain sizes $8 \pi$ and $3 \pi$ along the streamwise and spanwise directions. The Fourier modes used to construct the field of perturbations are integral multiples of the fundamental wavenumbers $k_{x, 0}=(2 \pi) /(8 \pi)$ for streamwise and $k_{y, 0}=$ $(2 \pi) /(3 \pi)$ for spanwise directions. The modes are truncated so that higher wavenumbers that are excluded have less than $0.001 \%$ of the energy in the most energetic Fourier mode; the number of Fourier modes needed to meet this truncation criterion goes from $(160,192)$ modes along streamwise and spanwise directions (counting positive and negative wavenumbers) at early times to $(40,40)$ modes at later times. Along the wallnormal direction, the Chebyshev collocation method is used.

To facilitate consistent discretization, the wall-normal Dirac delta function is approximated by the exponential function,

$$
\delta\left(z-z_{0}\right) \approx f_{s 0}\left(z ; z_{0}\right):=\frac{K}{2 \sqrt{\pi \epsilon}} \exp \left(-\frac{\left(R e_{\tau}\left(z-z_{0}\right)\right)^{2}}{4 \epsilon}\right),
$$

where $z_{0}$ is the location of the impulse, and $\epsilon$ quantifies the width of the impulse. We use $\epsilon=z_{0}^{+} / 4$ to have consistent discretization of the exponential function in eq. 2.7 near the wall and in the core of the channel; here, $z^{+}=R e_{\tau}(1+z)$ is the wall-normal coordinate expressed in inner units and referenced from the bottom wall, so that the bottom wall is at $z=-1$ and $z^{+}=0$. The constant $K$ in eq. (2.7) is set so that the function has an area of unity. The number of Chebyshev nodes used in the wall-normal direction is 768 ; increasing this number to 1152 produces a relative change in energy of less than $0.01 \%$ for all Fourier modes and times considered here.

The operators $A, B$, and $C$ have been validated against the results of Pujals et al. (2009) and Hwang \& Cossu (2010). The impulse response was separately validated about a laminar base flow against the results in chapter 10 of the $\mathrm{PhD}$ thesis of Jovanović (2004).

\section{Results}

The response to impulses introduced at three different locations, $z^{+} \approx 30, z^{+} \approx 500$, and $z=-0.5$ (or $z^{+}=5000$ ) at $R e_{\tau}=10000$ were computed at times $t u_{\tau} / h$ from 0.05 to 1.5 in steps of 0.05 , or $t U_{C L} / h$ from 1.42 to 41.2 in steps of 1.42. At each location, the body force is introduced along each of the three coordinate axes, producing a total of nine cases. For convenience, these cases shall be identified with a name such as I30x, where the 'I30' part refers to the forcing location being 30 inner units, and ' $x$ ' refers to the forcing direction. The names for the first six cases shall start with 'I30' or 'I500', followed by the direction of the forcing, while the last three cases shall start with 'O05' followed by the direction.

The topology of the coherent structures produced by the impulse response at different times is illustrated in fig. 1. where isosurfaces of swirling strength and streamwise velocity are plotted for I30x, I30y, and I30z cases for 8 different times: $t U_{C L} / h$ from 1.42 to 41.2 in steps of 5.7. The same isosurfaces are shown in fig. 2 for the I500 and O05 cases, but at only one time instant, $t U_{C L} / h=24.1$. The swirling strength is defined at each point in the domain as the imaginary part of the complex eigenvalue of the velocity gradient tensor (Zhou et al. 1999). The coherent structure produced by the 

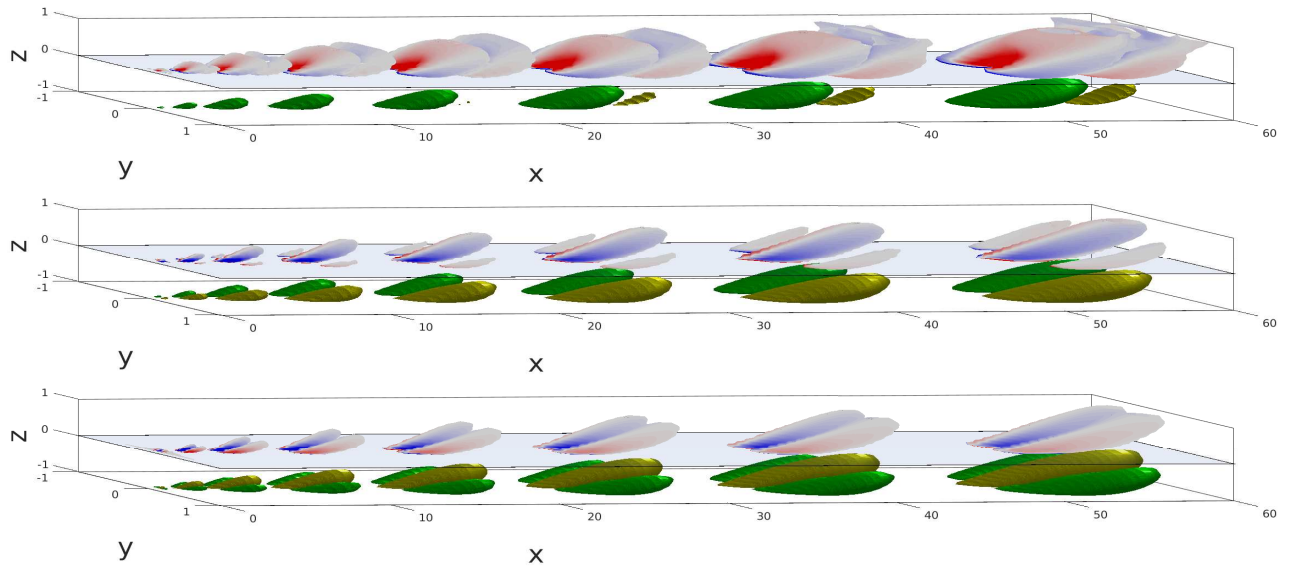

Figure 1. Coherent structures at times (from left to right) $t U_{C L} / h$ from 1.42 to 41.2 in steps of 5.7 due to the impulses (from top to bottom) I30x, I30y, and I30z. Green-yellow isosurfaces are for streamwise perturbation velocity at $+25 \%$ of instantaneous maximum (green) and $-25 \%$ of instantaneous maximum (yellow), with their streamwise locations modified to facilitate visualization. Red-white-blue isosurfaces are for swirling strength at $10 \%$ of the instantaneous maximum (colored by spanwise vorticity), and are plotted with a wall-normal offset of 1 to avoid overlap with the streamwise velocity isosurfaces.
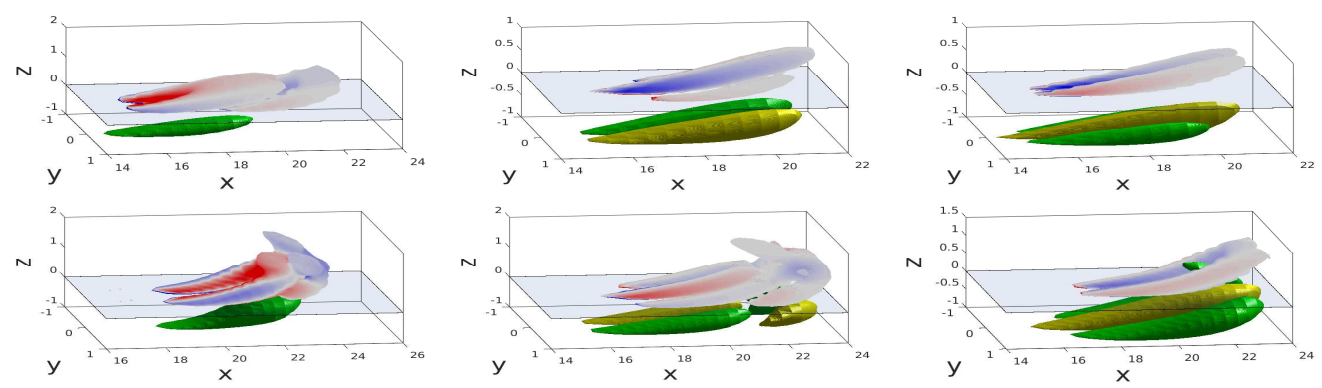

Figure 2. Coherent structures due to the impulses at $z^{+}=500$ (top) and $z=-0.5$ (bottom) along directions $x$ (left), $y$ (middle), and $z$ (right) at time $t U_{C L} / h=24.1$. Isosurfaces are as in fig. 1. and swirling strength has a wall-normal offset of 1 as before to avoid overlap with velocity isosurfaces.

impulse contains streamwise velocity streaks which are flanked or enclosed by quasistreamwise vortices. The coordinate axes are scaled unequally to facilitate visualization; the streamwise size of the structures is about 10 times longer than their wall-normal or spanwise size. The topology of the streaks and vortices change when the direction of the body force is changed, but changing the location from $z^{+} \approx 30$ to $z^{+} \approx 500$ does not affect a significant change. Forcing at $z=-0.5$ produces a more observable change in the structure of the perturbations, especially when the structures extend into the top half of the channel; however, features such as the number of streaks and vortices, and their relative arrangement, are retained. All of the structures also seem to be attached to the wall; this becomes more clear when Reynolds stresses are plotted. Geometric selfsimilarity is nominally observed in the evolution of these vortex-structures.

The self-similarity of the coherent structures can be seen more clearly by projecting isosurfaces of streamwise velocity (at $25 \%$ of instantaneous maximum) to the $x-z$ and $x-y$ planes, illustrated in fig. 3 for the I30x case, which has a single dominant streak. 

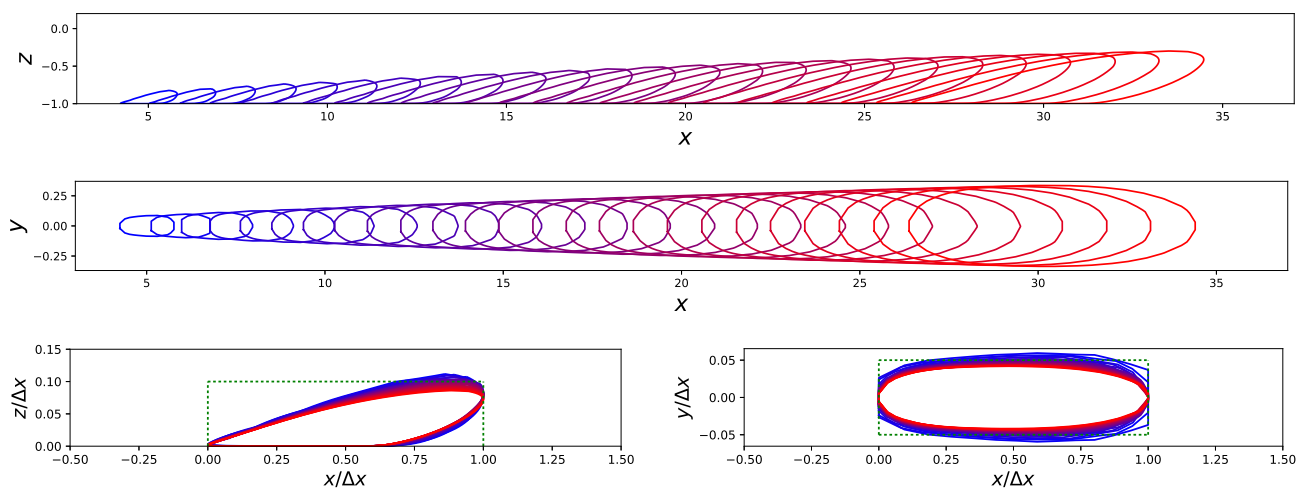

Figure 3. Isocontours of streamwise velocity (25\% of maximum) projected on the $y=0$ plane (top) and on the $z=0$ plane (middle) at times $t U_{C L} / h$ from 8.52 to 42.7 in steps of 1.42; figures on the bottom show these contours scaled by streamwise size $(\Delta x)$ and shifted to $x=0$, projected on $y=0$ (bottom left) and $z=0$ (bottom right). Contours are colored to show evolution in time in the overlaid contours in the bottom plots. Rectangles of aspect ratio 10 are also plotted (dotted lines) to highlight the aspect ratio of the structures.

These projections show an approximate collapse in rectangular boxes of aspect ratio 10 (also shown in fig. 3).

The self-similarity is further probed by considering the Reynolds stresses scaled by the height of the coherent structures (distance from the wall). For the periodic domain, the Reynolds stresses are defined as

$$
I_{i j}\left(\frac{1+z}{\delta_{h}}, t\right):=\frac{K}{u_{\tau}^{2}} \frac{k_{x, 0}}{2 \pi} \frac{k_{y, 0}}{2 \pi} \int_{x=0}^{2 \pi / k_{x, 0}} \int_{y=0}^{2 \pi / k_{y, 0}} u_{i}\left(x, y, \frac{1+z}{\delta_{h}}, t\right) u_{j}\left(x, y, \frac{1+z}{\delta_{h}}, t\right) \mathrm{d} x \mathrm{~d} y
$$

Here, all wall-normal distances are referenced from the wall (hence the $(1+z)$ dependence). The distances are scaled by $\delta_{h}$, which represents the wall-normal height of the coherent structure, and is defined as the maximum height from the bottom wall of the $|u|=0.25|u|_{\max }$ isosurface. The height of the structure is set to 1 whenever the isosurface extends into the top half of the channel. The constant $K$ is chosen such that the maximum of $-I_{13}$ is 1 . This scaling of the Reynolds stresses is similar to the eddy intensity functions of Townsend (1976), which is reasonable considering that the coherent structures in the impulse response are reminiscent of the attached eddies in Townsend's attached eddy hypothesis.

Reynolds stresses are shown for the nine cases of impulsive forcing in fig. 4. For all cases except O05x, the coherent structures have non-zero streamwise energy at $z^{+} \sim 10$ (shown in inset), with $I_{11} / \max \left(I_{11}\right)$ being greater than $10 \%$, thus showing that they are all attached to the wall. The shear intensity, $I_{13}$, is consistently negative, except for very tall structures with $\delta_{h}>0.5$ in the core region of the channel. The locations of the peaks of $I_{13}$ show good collapse, and this peak is located further away from the wall than the peak of $I_{11}$. The peaks of $I_{11}$ also show good collapse in the peak locations, but not in the peak magnitudes. The similarities in these three aspects - wall-attachedness, negative $I_{13}$, and the relative location of peaks of $I_{13}$ and $I_{11}$ - amongst the different impulse cases demonstrates that there is an underlying structure to the topology of the vortex-streak structures.

While the coherent structures exhibit self-similarity, they also decay quickly as shown 

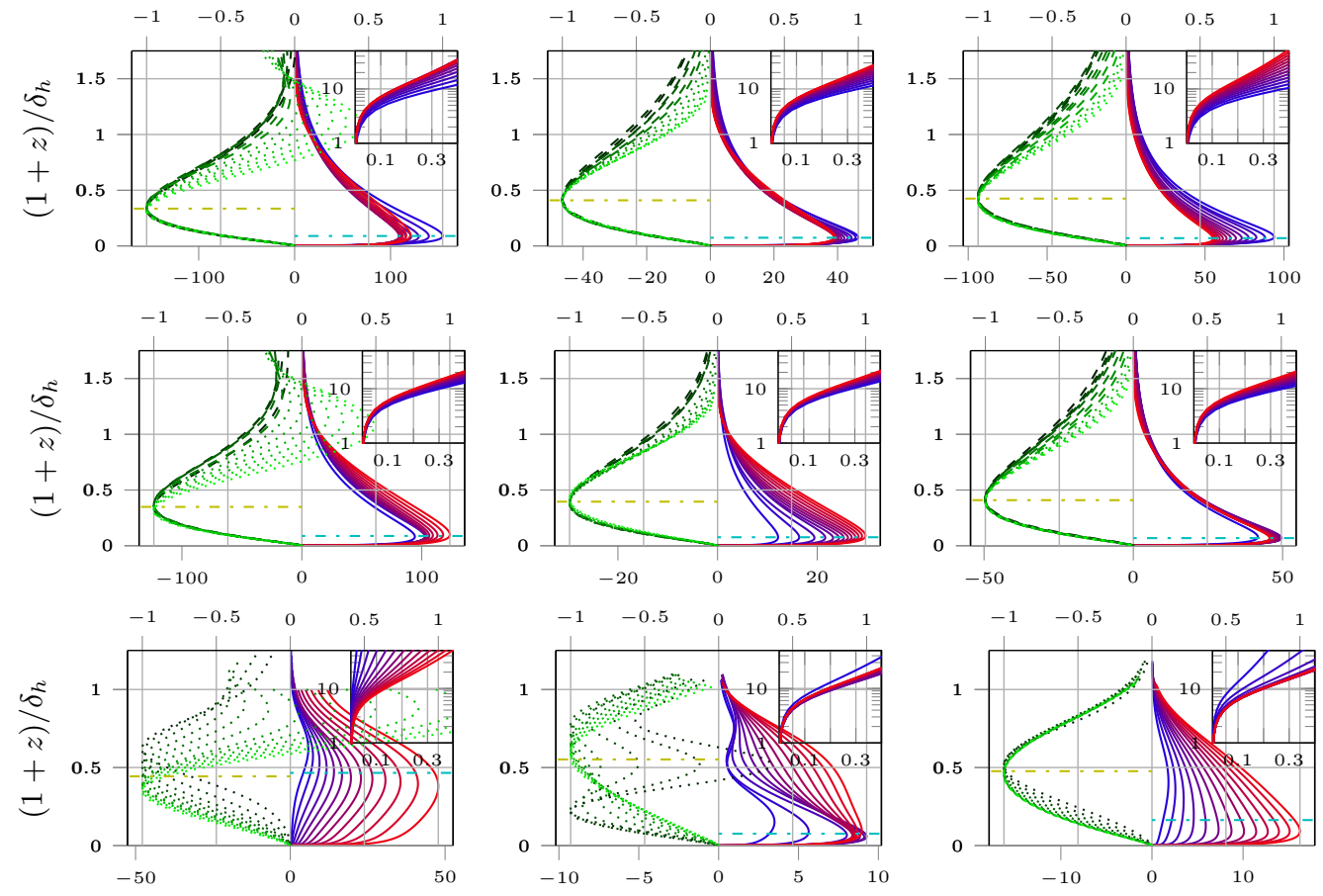

Figure 4. Reynolds stresses for impulses at $z^{+}=30$ (top row), $z^{+}=500$ (middle row), and $z=-0.5$ (bottom row) with forcing along $x$ (left columns), $y$ (middle columns), and $z$ (right columns) at times $t U_{C L} / h$ from 5.7 to 42.7 in steps of $2.84 ; I_{11}$ with scale on the bottom $x$-axis: blue to red for increasing time, $-; I_{13}$ with scale on the top $x$-axis: black to green for increasing time, - - for structures with $\delta_{h} \leqslant 0.5$ and ….. otherwise. The average locations of the peaks of $\left.I_{11}(-)^{-}\right)$and $I_{13}(--)$ are also shown. All curves are truncated to ignore the top half of the channel $(z>1)$. Insets show near-wall streamwise energy: $I_{11} / \max \left(I_{11}\right)$ on the $x$-axis against $z^{+}$on the $y$-axis in log scale; note that $z^{+}$here is not scaled by $\delta_{h}$.

in fig. 5. where the streamwise and total kinetic energy density are shown for all nine impulses. The kinetic energy density is defined for each velocity component, $E_{u_{i}}$, as

$$
E_{u_{i}}:=\frac{k_{x, 0}}{2 \pi} \cdot \frac{1}{2} \cdot \frac{k_{y, 0}}{2 \pi} \int_{x=0}^{2 \pi / k_{x, 0}} \int_{y=0}^{2 \pi / k_{y, 0}} \int_{z=-1}^{1}\left(\frac{u_{i}}{u_{\tau}}\right)^{2} \mathrm{~d} x \mathrm{~d} y \mathrm{~d} z,
$$

with the total kinetic energy density $E$ being their sum, $E=E_{u}+E_{v}+E_{w}$. For the I30 and 1500 cases, the energy in the perturbations reduces by a few orders of magnitude by the time the structures reach the core of the channel $\left(\delta_{h} \gtrsim 0.5\right)$. For the near-wall case, the total energy is predominantly due to streamwise motion for the range of times considered here. For the impulses further away from the wall, however, there is significant energy in the cross-stream components. As the location of the impulse moves further away from the wall, the energy in the cross-stream components remains significant quite late into the decay. We reason that these differences arise due to the local differences in the mean shear and the eddy viscosity; due to the large mean shear near the wall, crossstream velocities extract a large streamwise perturbation velocity from the mean velocity, while the cross-stream components themselves dissipate due to the eddy viscosity. For impulses away from the wall, the mean shear is significantly weaker, leading to slower extraction of streamwise velocity perturbation energy from the mean velocity. A detailed 

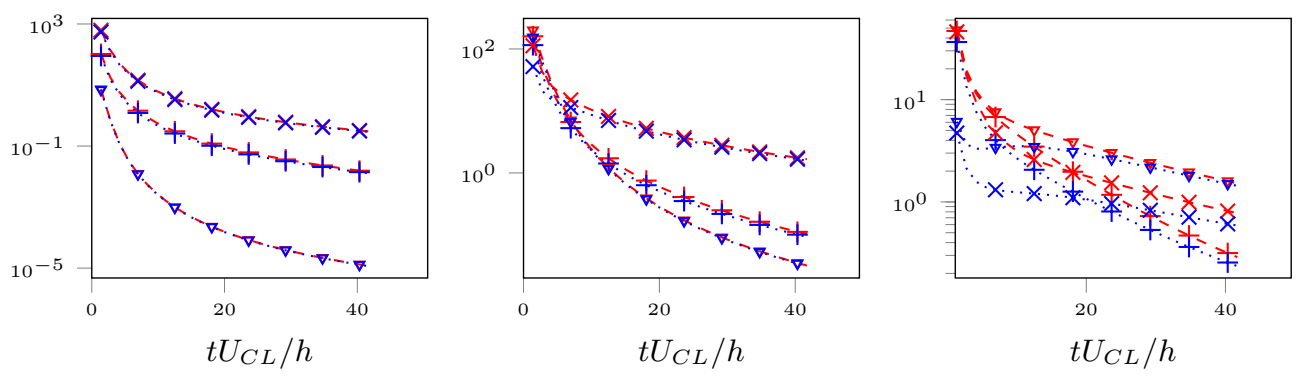

Figure 5. Evolution of total (- - ) and streamwise (......) kinetic energy density for impulses introduced at different locations: $z^{+} \approx 30$ (left), $z^{+} \approx 500$ (middle), and $z=-0.5$ (right). For each location, the plots show energy due to impulses along streamwise $(+)$, spanwise $(\times)$, and wall-normal $(\nabla)$ directions. Markers correspond to times where coherent structures are plotted in fig. 1 .

investigation of the early time evolution of these perturbations is needed to shed light on these mechanisms.

\section{Conclusion and outlook}

The impulse response of the eddy-viscosity-enhanced linearized Navier-Stokes equations (eLNSE) shows spatially growing vortex-streak structures that have approximate geometric self-similarity, illustrated here at $R e_{\tau}=10000$. The coherent structures produced here are quite similar to the vortex-streak structures known to be important to turbulence (Jiménez \& Moin 1991; Halcrow et al. 2009 , Hall \& Sherwin 2010; Hwang 2015). These structures have an aspect ratio (streamwise size to wall-normal size) of approximately 10, which is close to the experimental observations of aspect ratios of attached eddies in the log layer (reported to be $\approx 14$ based on linear coherence by Baars et al. (2017) for boundary layers). The topology of the vortex streak structure does not change when the forcing is moved from $z^{+} \approx 30$ to $z^{+} \approx 500$, but is dependent on the direction of the impulsive body force. This trend is also observed for forcing at $z=-0.5$, albeit with noticeable differences in the shape of these structures as they extend into the top half of the channel. The coherent structures due to all of the nine impulses considered here evolve to have non-zero energy extending down to $z^{+} \sim 10$, i.e., they are all attached to the wall. This includes the cases where the forcing is introduced at $z=-0.5$. The coherent structures for all cases also produce a negative Reynolds shear stress $I_{13}$, which peaks further away from the wall than $I_{11}$. These observations are consistent with the supposed prevalence of attached eddies and the significance of ejection and sweeping events in turbulence. In short, irrespective of the location and direction of the impulse, the resulting structures are vortex-streak structures that are wall-attached and self-similar with negative $I_{13}$ and an aspect ratio of approximately 10 . Considering this robust behaviour of the impulse response, we can expect that employing a spatio-temporally distributed body forcing would also produce similar structures. This provides more direct evidence, albeit under the approximation of the eLNSE model, for wall-attached hairpin-like vortices flanking velocity streaks in high Reynolds number flows.

The energy decay seen in the impulse response needs further clarification, since the large-scale structures appear to have very little energy density, which was also noted by Eitel-Amor et al. (2015). This hurdle could possibly disappear when, instead of response to isolated impulses, an appropriate sum of impulse responses is used; such forcing would 
represent the response to some spatio-temporally distributed forcing instead of a spatiotemporally localized forcing. Indeed, the Orr-Sommerfeld-Squire operator employed here is capable of producing energy growth that is larger than is needed to capture turbulence statistics (see fig. 16 of Zare et al. 2017). It is worth noting that the spatio-temporally distributed forcing that must be considered to compensate for the energy decay cannot be the actual Reynolds shear stresses, since a part of these stresses have been absorbed into the eddy viscosity. It would be interesting to determine such forcing, which would be an analogue of the work of Zare et al. (2017) for a flow-field decomposed into self-similar coherent structures instead of Fourier modes. Unfortunately, no numerical schemes are presently available, as far as the authors are aware, that could achieve this; further limitations arise from the increasing difficulty to obtain spatio-temporally resolved measurements with increasing Reynolds number.

An alternative approach to constructing turbulence fields with the present coherent structures is the attached eddy model of Perry \& Marusic (1995), which describes turbulent fluctuations as a superposition of self-similar attached eddies with a prescribed spatial distribution. The geometrically self-similar vortex-streak structures found in this work have the potential to be used as the building blocks in the attached eddy model; this would bypass the need to prescribe the spatio-temporal distribution of the forcing. However, preliminary calculations show (not included in this paper) that the ratio of the peaks of the streamwise kinetic energy to the negative of the shear stress, $\max \left(I_{11}\right) / \max \left(-I_{13}\right)$, due to the present coherent structures are higher, by a factor of order 10 , than are needed to match the slopes of the Reynolds stresses in a fully turbulent flow using the attached eddy model. This difference possibly suggests that the required attached eddy velocity field requires more than the linear response considered here. This could include modifications to the linear operator or the inclusion of non-linear interactions. However, these remain open issues that require further investigation. Even so, the robustness of the coherent structures found using the computationally cheap eLNSE model shows good potential in identifying a fundamental self-similar basis to describe turbulence dynamics.

\section{REFERENCES}

Adrian, R. J. 2007 Hairpin vortex organization in wall turbulence. Phys. Fluids 19 (4), 041301.

BaArs, W. J., Hutchins, N. \& Marusic, I. 2017 Self-similarity of wall-attached turbulence in boundary layers. J. Fluid Mech. 823, R2.

Cess, R. D. 1958 A survey of the literature on heat transfer in turbulent tube flow. Tech. Rep.. 8-0529-R24. Westinghouse Research.

Codrignani, A. R. 2014 Impulse response in a turbulent channel flow. PhD thesis, Politec. Milano.

Del Álamo, J. C. \& Jimenez, J. 2006 Linear energy amplification in turbulent channels. J. Fluid Mech. 559, 205-213.

Eitel-Amor, G., Örlü, R., Schlatter, P. \& Flores, O. 2015 Hairpin vortices in turbulent boundary layers. Phys. Fluids 27 (2), 025108.

Halcrow, J., Gibson, J. F., Cvitanović, P. \& Viswanath, D. 2009 Heteroclinic connections in plane couette flow. J. Fluid Mech. 621, 365-376.

Hall, P. \& Sherwin, S. 2010 Streamwise vortices in shear flows: harbingers of transition and the skeleton of coherent structures. J. Fluid Mech. 661, 178-205.

Henningson, D. S. \& Reddy, S. C. 1994 On the role of linear mechanisms in transition to turbulence. Phys. Fluids 6 (3), 1396-1398.

Hoyas, S. \& JimÉnEz, J. 2006 Scaling of the velocity fluctuations in turbulent channels up to $R e_{\tau}=2003$. Phys. Fluids 18 (1), 011702. 
HwANG, Y. 2015 Statistical structure of self-sustaining attached eddies in turbulent channel flow. J. Fluid Mech. 767, 254-289.

Hwang, Y. 2016 Mesolayer of attached eddies in turbulent channel flow. Phys. Rev. Fluids $1(6), 064401$.

Hwang, Y. \& Cossu, C. 2010 Linear non-normal energy amplification of harmonic and stochastic forcing in the turbulent channel flow. J. Fluid Mech. 664, 51-73.

Jiménez, J. \& Moin, P. 1991 The minimal flow unit in near-wall turbulence. J. Fluid Mech. 225, 213-240.

Jovanović, M. \& Bamien, B. 2001 The spatio-temporal impulse response of the linearized Navier-Stokes equations. In Proc. 40th IEEE Conf. Decision and Control, pp. 1948-1953. Orlando, FL.

Jovanović, M. R. 2004 Modeling, analysis, and control of spatially distributed systems. PhD thesis, University of California at Santa Barbara.

Kim, J. \& Lim, J. 2000 A linear process in wall-bounded turbulent shear flows. Phys. Fluids $12(8), 1885-1888$.

Landahl, M. T. 1990 On sublayer streaks. J. Fluid Mech. 212, 593-614.

Luchini, P., Quadrio, M. \& Zuccher, S. 2006 The phase-locked mean impulse response of a turbulent channel flow. Phys. Fluids 18 (12), 121702.

Mathis, R., Hutchins, N. \& Marusic, I. 2009 Large-scale amplitude modulation of the small-scale structures in turbulent boundary layers. J. Fluid Mech. 628, 311-337.

McKeon, B. J. 2017 The engine behind (wall) turbulence: perspectives on scale interactions. J. Fluid Mech. 817, P1.

McKeon, B. J. \& Sharma, A. S. 2010 A critical-layer framework for turbulent pipe flow. J. Fluid Mech. 658, 336-382.

Moarref, R., Sharma, A. S., Tropp, J. A. \& McKeon, B. J. 2013 Model-based scaling of the streamwise energy density in high reynolds number turbulent channels. J. Fluid Mech. 734, 275-316.

Perry, A. E. \& MArusic, I. 1995 A wall-wake model for the turbulence structure of boundary layers. part 1. extension of the attached eddy hypothesis. J. Fluid Mech. 298, 361-388.

Pujals, G., García-Villalba, M., Cossu, C. \& Depardon, S. 2009 A note on optimal transient growth in turbulent channel flows. Phys. Fluids 21 (1), 015109.

Reynolds, W. C. \& Hussain, A. K. M. F. 1972 The mechanics of an organized wave in turbulent shear flow. part 3. theoretical models and comparisons with experiments. J. Fluid Mech. 54 (2), 263-288.

Reynolds, W. C. \& Tiederman, W. G. 1967 Stability of turbulent channel flow, with application to malkus's theory. J. Fluid Mech. 27 (2), 253-272.

Townsend, A. A. 1976 The structure of turbulent shear flow. Cambridge University Press.

WALEFfE, F. 1995 Transition in shear flows. nonlinear normality versus non-normal linearity. Phys. Fluids 7 (12), 3060-3066.

Zare, A., Jovanović, M. R. \& Georgiou, T. T. 2017 Colour of turbulence. J. Fluid Mech. 812, 636-680.

Zhou, J., Adrian, R. J., Balachandar, S. \& Kendall, T. M. 1999 Mechanisms for generating coherent packets of hairpin vortices in channel flow. J. Fluid Mech. 387, 353396. 